\title{
Pneumoretroperitoneum after Attempted Epidural Anesthesia
}

\author{
Arkadi Isakov ${ }^{*}$, Arnold Shtein², Sholmo Kyzer $^{1}$ \\ 1 Department of Surgery "B," Wolfson Medical Center, Holon, Sackler Faculty of Medicine, Tel Aviv University, Tel Aviv, \\ Israel \\ 2 Department of Anesthesiology, Wolfson Medical Center, Holon, Sackler Faculty of Medicine, Tel Aviv University, Tel \\ Aviv, Israel
}

\begin{abstract}
Air may extend to the retroperitoneal space from retroperitoneal perforation of a hollow viscus, infection of the anterior pararenal space with gas-forming organisms and from pneumothorax or pneumomediastinum [1]. Rare pathologies, such as open reduction and internal fixation of femoral fractures and anaerobic abscess of the hip joint have also been described in relation to this complication [1,2]. A rare case of pneumoretroperitoneum caused by insufflation of air during an attempt to achieve epidural anesthesia is described.
\end{abstract}

Keywords: pneumoperitoneum, anaesthesia, epidural

Received: 04 August 2016 / Accepted: 15 September 2016

\section{CASE REPORT}

An 81-year-old, Caucasian, male, weight $72 \mathrm{~kg}$, height $178 \mathrm{~cm}$, was admitted due to fracture of the neck of the right femur. The patient was operated on, and internal fixation of the fracture was performed. Epidural anesthesia at the L4-5 level in the right the lateral position was performed by a second-year anesthesia resident. A midline approach and an 18G Tuohy needle were used. The procedure was technically difficult, and a total of three attempts were made. Loss of resistance with air was used. During the efforts to identify the epidural space, some 30 to 35 milliliters of air had been injected. Subjective loss of resistance was reported by the performing anesthesiologist. However, he was unable to insert an epidural catheter. Local anesthetic was not injected. The procedure was eventually carried out without intraoperative complications under general anesthesia. On the fourth postoperative day, the patient developed continuous abdominal pain of moderate severity. On examination, there were no signs of peritoneal irritation. However, an abdominal CT scan demonstrated air in the retroperitoneum around the left psoas muscle (Figure 1). Because the patient had received an enema on the third postoperative day, a gastrografin enema was performed to rule out rectal or colonic perforation. No leak was demonstrated. CT examination confirmed air around the left psoas muscle which extended to the left paravertebral muscles. There was no air around the pelvic organs or at the site of the femoral fixation. The patient's condition improved gradually, and he was discharged on the seventh postoperative day. The patient agreed with presentation of his data and all the procedures performed were approved by the institution where the patient was treated.

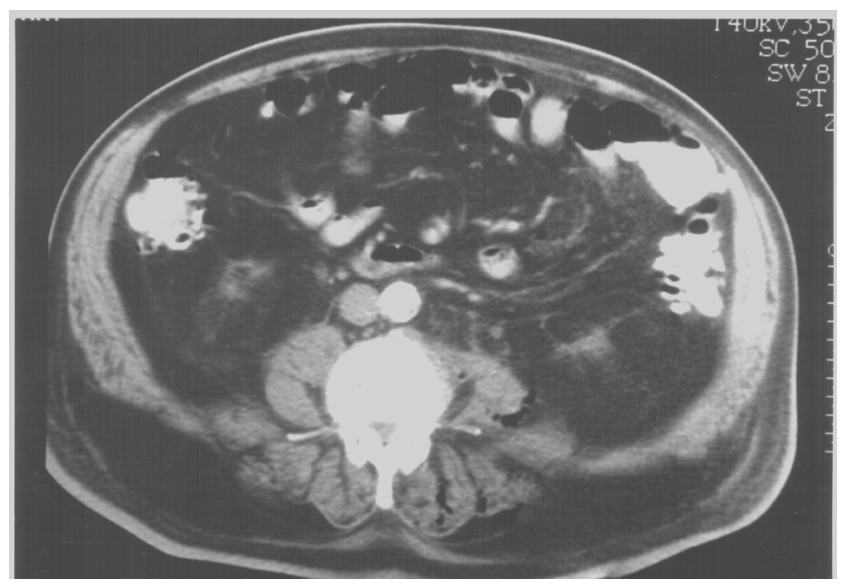

Fig. 1. Abdominal CT scan. Air around the left psoas muscle 


\section{DISCUSSION}

The presence of air in the retroperitoneum and its early recognition and detection of the source are important, to rule out an unobserved serious cause [1].It is also imperative to recognize the cases in which pneumoretroperitoneum is not caused by perforation of the retroperitoneal hollow viscus. Recognition of these cases may prevent unnecessary laparotomies.

Maldjian and Misbaun described a young patient who developed pneumoretroperitoneum after open reduction and internal fixation of a proximal femoral fracture [2].The largest amount of air was located in the deep soft tissues at the site of the femoral fixation and the pelvis. In our case, retroperitoneal air was not present at the pelvis or around the location of the femoral fixation. However, air was demonstrated to have extended to the paravertebral tissues. Due to the location of the air and the benign course of the case, we speculated, that the pneumoretroperitoneum in this patient was caused by the attempts to achieve epidural anesthesia which included air insufflation.

The most probable cause for the presence of air around the psoas muscle in the retroperitoneum is the faulty identification of the psoas compartment as the epidural space. While performing the psoas compartment block for unilateral lumbar anesthesia, the loss of resistance technique, similar to that used for an epidural block, is employed as described by Chayen and Winnie $[3,4]$.Although the actual anatomical existence of this compartment is debated by others, a distinct loss of resistance is felt when the epidural needle penetrates the anterior fascia of the quadratus lumborum muscle $[5,6]$.

When the needle is inserted perpendicular to the skin and using a straight paramedian path, the psoas compartment can usually be found at a depth of approximately $7 \mathrm{~cm}$ from the entry point [7].However, from a midline entry point, if a needle is inserted at an angle, the distance to the compartment would be 1 to $2 \mathrm{~cm}$ longer. In the presented case, CT imaging showed air in the space between the psoas and the quadratus lumborum. Such a faulty identification of the psoas compartment is possible if the epidural needle was inserted at an angle off midline so that the tip would end up laying at a maximum depth of 10 to $11 \mathrm{~cm}$ and 3 to $5 \mathrm{~cm}$ lateral to the midline from the skin entry point. Not surprisingly, the epidural block was unsuccessful. Furthermore, a lumbosacral block does not provide sufficient anesthesia for hip surgery in the majority of patients [7-11].

It is most unlikely that a hollow viscus can be perforated by an epidural needle during the procedure. Given the limited needle length $(10 \mathrm{~cm})$ and the average size of a patient, the chance of reaching and penetrating the peritoneum is low. Furthermore, even if a needle did approach the bowel wall, owing to the rounded tip of a Tuohy needle, it would most likely push the bowel away instead of piercing it. Furthermore, if the bowel had been perforated, the probability is that a retroperitoneal abscess or intraperitonial air would develop. The possibility that the air had tracked cephalad from the site of an open reduction of femur can be rejected, because the air was found at the opposite side to the surgical site.

We cannot provide a convincing explanation of how the air in the retroperitoneum causes pain. Tracking through fascial sheaths, irritation of nerves and tissue distention are possible mechanisms. Air bubbles can be found to persist in the epidural or subararachnoid space for days after epidural block with air. In patients with subcutaneous or eyelid emphysema, the absorption of air could last several days up to two weeks [12]. One would expect, however, that the amount of air should be significantly decreased four days after surgery. Thus, even if blame is attributed to air found in the psoas compartment, as a cause for the patient's complaints, these would be expected to occur earlier rather than later.

Finding air was incidental to the evaluation of postoperative abdominal pain. The pain was eventually relieved after the gastrographin enema and subsequent bowel movement.

The aim of this case presentation is to alert against the use of large amounts of air during the performance of epidural anesthesia. Retroperitoneal air is only one of the potentially dangerous complications associated with this practice. This issue was extensively addressed by a recent review by Saberski et al [13]. One of the easiest methods to avoid this complication is by using fluid instead of air for loss of resistance during identification of the epidural space.

Because epidural anesthesia is widely used, it is very important for surgeons, anesthesiologists, and radiologists to be aware of the association, although rare, between attempts for epidural anesthesia and pneumoretroperitoneum. Recognition of this association may prevent unnecessary operative interventions. 


\section{CONFLICT OF INTEREST}

\section{Nothing to declare}

\section{REFERENCES}

1. Pretre R, Robert J, Mirescu D, Witzig JA, Rohner A. Pathophysiology, recognition and management of pneumoretroperitoneum. Br J Surg. 1993;80:1138-40.

2. Maldjian PD, Nusbaum AO. Pneumoretroperitoneum secondary to an open reduction and internal fixation of a femoral fracture: Case report. Am Surg. 1997;63:504-5.

3. Chayen D, Nathan H, Chayen M. The psoas compartment block. Anesthesiology. 1976;45:95-9.

4. Winnie AP, Ramamurthy S, Durrani Z, Radongic K. Plexus blocks for low extremity surgery. Anesthesiology Review. 1974;1:11-6.

5. Hanna MH, Peat SJ, D’Costa F. Lumbar plexus block: anatomical study. Anaesthesia. 1983;48:675-8.

6. Chudinov A, Berkenstadt $H$, Salai $M$, Kahaha A, Pearl A. Continuous psoas compartment block for anaesthesia and perioperative analgesia in patients with hip fractures. Regional Anesthesia and Pain Management. 1999;24:563-8.
7. Kirshmair L. Ultrasound guided psoas compartment block: verification of a new technique with $\mathrm{CT}$. The International Monitor. 2000;12:199.

8. Parkinson SK, Mueller GB, Little WL, Bailly SL. Extent of blockade with various approaches to the lumbar plexus. Anesth Analg. 1989;68:243-8.

9. Farny J, Gerrard M, Drolet P. Posterior approach to the lumbar plexus combined with sciatic nerve block using lidocaine. Can J Anaesth. 1994;41:486-91.

10. White I, Chappell WA. Anaesthesia for surgical correction of fractured femoral neck - a comparison of three techniques. Anaesthesia. 1980;35:1107-10.

11. Barret JB, Mostarlic O. Psoas compartment block. Anaesthesia. 1986;41:1058-59.

12. Alvares JF, Dhawan PS, Tibrewala S, et al. Retroperitoneal perforation in ulcerative colitis with mediastinal and subcutaneous emphysema. J Clin Gastroenterol. 1997;25:4535.

13. Saberski LR, Kondamuri S, Osinubi OY. Identificatoin of the epidural space: is loss of resistance to air a safe technique? A review of the complications related to the use of air. Reg Anesth. 1997;22:3-15. 